\title{
CircRNA expression profile and functional analysis in testicular tissue of patients with non-obstructive azoospermia
}

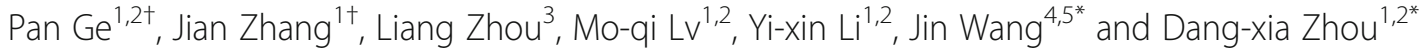

\begin{abstract}
Background: Non-obstructive azoospermia (NOA) is a multifactorial disorder whose molecular basis remains largely unknown. Circular RNAs (CircRNAs), a novel class of endogenous RNAs, have been recognized to play important roles in many biological processes. However, little is known about the expression patterns and functions of circRNAs in human testes involved in NOA.
\end{abstract}

Methods: In this study, the testicular circRNA expression profile were explored in NOA patients and the controls by high-throughput circRNA microarray. Real-time quantitative reverse transcription polymerase chain reaction (qRTPCR) was performed to confirm the microarray data. Bioinformatics analyses including the circRNA/miRNA/mRNA interaction network, Gene Ontology (GO) and Kyoto Encyclopedia of Genes and Genomes (KEGG) pathway analysis were used to predict the functions of differentially expressed circRNAs.

Results: A total of 368 differentially down-regulated and 526 up-regulated circRNAs were detected in NOA patients. These findings have been verified by qRT-PCR on 6 selected circRNAs. Among these differentially expressed circRNAs, the hsa_circRNA_0023313 was obviously up-regulated in testicular tissue of NOA patients. The most likely potential target miRNA for hsa_circRNA_0023313 include hsa-miR-520d-3p, hsa-miR-373-3p, hsa-miR-372-3p, hsamiR-302c-3p and hsa-miR-130b-5p. Function analysis indicated that hsa_circRNA_0023313 was ubiquitin-protein transferase activity and chromatin binding. KEGG analysis revealed that the top five pathways related to hsa_ circRNA_0023313 were endocytosis, meiosis, FoxO signaling pathway, ubiquitin mediated proteolysis and AMPK signaling pathway.

Conclusions: This is the first report that the testicular circRNA expression profile is altered in NOA patients indicating circRNAs might play important roles in regulating spermatogenesis and be potential biomarkers for the diagnosis and treatment of NOA.

Keywords: CircRNA, Spermatogenesis, Non-obstructive azoospermia (NOA), Microarray, Bioinformatics analyses

\section{Background}

Infertility is a worldwide reproductive health problem that affects an estimated 70 million people globally [1]. The world Health Organization estimate that $10-15 \%$ of couples struggle with infertility issues and male factors account for about half of all infertility cases $[2,3]$

\footnotetext{
*Correspondence: 253299433@qq.com; zdxtougao@163.com

${ }^{\dagger}$ Pan Ge and Jian Zhang contributed equally to this work.

${ }^{4}$ Obstetrics and Gynecology Department, Xi'an Angel Women's \& children's Hospital, Xi'an 710077, China

'Department of Pathology, Medical School, Xi'an Jiaotong University, Xi'an 710061, China

Full list of author information is available at the end of the article
}

Unfortunately, nearly $60-75 \%$ of male infertility is unexplained or idiopathic, since the molecular mechanisms underlying the defects remain unknown $[4,5]$. Nonobstructive azoospermia (NOA) is the most severe manifestation of male infertility which spermatogenesis process is disrupted [6, 7], it affects $1 \%$ of males and $10 \%$ of those who seek fertility assistance [8]. It also demonstrated that NOA accounts for approximately $60 \%$ azoospermia in which spermatogenesis process is inactive and thus sperm cells are not generated [9]. Up to now, NOA is a multifactorial disorder whose molecular basis remains largely unknown [6, 10]. Although

(C) The Author(s). 2019 Open Access This article is distributed under the terms of the Creative Commons Attribution 4.0 International License (http://creativecommons.org/licenses/by/4.0/), which permits unrestricted use, distribution, and 
microdissection testicular sperm extraction (microTESE) is the standard therapy for NOA, sperm retrieval is unsuccessful in approximately $50 \%$ of patients [11]. Therefore, the challenge is to elucidate the precise molecular mechanisms involved in spermatogenesis process and to discover the effective diagnostic markers or therapeutic targets for NOA patients.

Circular RNAs (CircRNAs) are a novel type of endogenous RNAs featuring stable structure and high tissue-specific expression [12]. Unlike linear RNAs, circRNAs form a covalently closed continuous loop, which allows circRNAs to resist the degradation and are highly represented in the eukaryotic transcriptome [13]. CircRNAs are much more stable and conserved than linear RNAs and therefore might be involved in more abundant functions. Research has revealed that circRNAs can function as miRNA sponges, regulators of splicing and transcription, and modifiers of parental gene expression [14]. CircRNAs have been considered important biological regulators for understanding the molecular mechanisms of disease and identifying effective diagnostic biomarkers or therapeutic targets [15]. Recently, circRNAs are reported to be involved in the development of many diseases such as cardiovascular diseases and various cancers [15-19]. However, so far, to our knowledge, little is known about the expression and function of circRNAs in male infertility.

Therefore, the current study aimed to investigate the expression profile and functions of circRNAs in NOA patients. Bioinformatics analysis were also used to identify the circRNA/miRNA/mRNA interaction network, biological process and signal pathways. These results may provide potential targets for the development of novel diagnostic and therapeutic strategies against NOA.

\section{Materials and methods \\ Patients and samples}

The protocol was fully approved by the Institutional Medical Ethics Committee of Xi'an Jiaotong University. The purpose of this study was explained to all subjects, and written informed consent forms were obtained from all subjects. NOA patients were selected from couples attending the infertility clinic in reproductive center of Northwest women and children Hospital who had a history of infertility of $\geq 12$ months. Three times semen analyses were carried out after 3-7 days of sexual abstinence. Patients with chronic diseases, hypoandrogenism, hypogonadism, history of pelvic/spinal injuries, karyotype abnormalities and microdelections of AZF region on $\mathrm{Y}$ chromosome were excluded. According to the World Health Organization (WHO) 2010 guidelines, all NOA patients were diagnosed by detecting three times semen samples without spermatozoa in the ejaculate including high-speed centrifugation of the entire pellet [20-23].

Finally, testicular samples were obtained from 50 patients with NOA (ages 25-46 years). An ideal normal control should consist of volunteers of known fertility, but difficulties in acquiring testicular samples makes it impractical. Therefore, 50 patients (ages 25-40 years) with obstructive azoospermia (OA) whose testicular histopathological examination demonstrated normal spermatogenesis were used as controls. Of which, 3 NOA patients whose testicular histopathological examination showed early maturation arrest and 3 controls were further used for circRNA microarray labeling and hybridization.

\section{RNA extraction and quality control}

Total RNA was extracted from testicular biopsy tissues with TRIzol reagent according to the manufacturer's instructions (Invitrogen, Carlsbad, California, USA). In order to reduce the inter-group difference, we mixed the three testicular tissue samples in NOA and the control group respectively for subsequent circRNA microarray labeling and hybridization. The RNA quantification and quality was examined by using the Nanodrop ND-1000 spectrophotometer. RNA integrity and gDNA contamination was tested by denaturing agarose gel electrophoresis.

\section{CircRNA microarray labeling and hybridization}

The sample preparation and microarray hybridization were performed based on the Arraystar's standard protocols provide by KANGCHENG Inc. (Shanghai, China). Firstly, total RNAs of 2 groups were digested with Rnase R (Epicentre, Inc.) to remove linear RNAs and enrich circular RNAs respectively. Secondly, the enriched circular RNAs were amplified and transcribed into fluorescent cRNA utilizing a random priming method (Arraystar Super RNA Labeling Kit; Arraystar). Thirdly, the labeled cRNAs were hybridized onto the Arraystar Human circRNA Array (8x15K, Arraystar). Finally, after having washed the slides, the arrays were scanned by the Agilent Scanner G2505C.

\section{Microarray data collection and analysis}

Briefly, acquired array images were analyzed by using Agilent Feature Extraction software (version 11.0.1.1). Quantile normalization and subsequent data processing were performed using the $\mathrm{R}$ software package. Differentially expressed circRNAs with statistical significance between two groups were explored by Scatter Plot filtering. Differentially expressed circRNAs between samples were identified through Fold Change filtering. Hierarchical Clustering was performed to show the distinguishable circRNAs expression pattern among samples. 


\section{Validation of circRNA by qRT-PCR}

Real-time quantitative reverse transcription polymerase chain reaction (qRT-PCR) was performed to confirm the circRNA microarray data. 6 differentially expressed circRNAs (including 3 up-regulated and 3 down-regulated) were selected for qRT-PCR experiments in 50 pairs of fresh frozen testicular tissue samples (50 from NOA and 50 from OA). Specific primers designed for circRNAs were listed in Table 1 . The primers were synthesized by Tsingke Biotech Ltd. (Beijing, China).

Firstly, total RNA from testicular samples was prepared using MiniBEST Universal RNA Extraction Kit (Takara, Japan) according to the manufacturer's protocol. Secondly, total RNA was reverse transcribed into cDNA by using the HiFiScript cDNA Synthesis Kit (CWBIO, China) in a $20 \mu$ reaction volume. Thirdly, real time PCR was performed on the Bio CFX Connect realtime PCR analyzer (Bio-Red, USA) by using the UltraSYBR Mixture (High ROX) (CWBIO, China). In brief, the total volume of $10 \mu \mathrm{l}$ PCR reactions was prepared by mixing $5 \mu \mathrm{l}$ UltraSYBR Mixture $(2 \times), 0.3 \mu \mathrm{l}$ each forward and reverse primer and $10 \mathrm{ng}$ cDNA. The reaction conditions were as follows: initial incubation at $95^{\circ} \mathrm{C}$ for 10 min, followed by 40 cycles of $10 \mathrm{~s}$ denaturation at $95^{\circ} \mathrm{C}$, 30 s annealing at $57^{\circ} \mathrm{C}$ and $32 \mathrm{~s}$ extension at $72{ }^{\circ} \mathrm{C}$. All of the experiments performed in triplicate, and the average $\mathrm{Ct}$ value was used to calculate the relative expression of circRNA through the comparative $2^{-\Delta \Delta \mathrm{Ct}}$ method.

\section{CircRNA/miRNA interaction and circRNA/miRNA/mRNA regulatory networks analysis}

To identify the potential functions of selected circRNAs, the circRNA/miRNA interaction was predicted using Arraystar's home-made miRNA target prediction

Table 1 Primer sequences

\begin{tabular}{lllc}
\hline CircBase ID. & F/R & Primer Sequence & size (bp) \\
\hline hsa_circ_0058058 & F & 5'-GCAGAGCTCCGAGAGTAAGG-3' & 89 \\
& R & 5'-AGGCCGGTTTGTCAGAGAC-3' & \\
hsa_circ_0008045 & F & 5'-GAGCCAGGACAAGACTCTCAA-3' & 97 \\
& R & 5'-ATTCAGCAGTTGGATGCCGA-3' & \\
hsa_circ_0023313 & F & 5'-AAAACGCTACCTCGCTGCAC-3' & 143 \\
& R & 5'-GGCCTCTGCTTCGCTGAT-3' & \\
hsa_circ_0061817 & F & 5'-ACTGGTGAGGAACATCCACG-3' & 91 \\
& R & 5'-AAGAACTGAATAGCCTGGCCC-3' & \\
hsa_circ_0002023 & F & 5'-CCAGCCCCAAAGAGTCAACTAA-3' & 164 \\
& R & 5'-TCCATCGAGAAGGTCCACGAA-3' & \\
hsa_circ_0008533 & F & 5'-CACACCTGGACAGTCAGTTCT-3' & 125 \\
& R & 5'-ATCAGCTCCTCCAGCTCATCT-3' & \\
GADPH & F & 5'-GCACCGTCAAGGCTGAGAAC-3' & 138 \\
& R & 5'-TGGTGAAGACGCCAGTGGA-3' & \\
\hline
\end{tabular}

software based on miRanda [24] and TargetScan (http:// www.targetscan.org) [25]. The differentially expressed circRNA were annotated in detail using the circRNA/ miRNA interaction information. In addition, the circRNA/miRNA/mRNA regulatory networks were further predicted according to the target genes of circRNA targeting miRNAs by starBase v2.0 (http://starbase.sysu. edu.cn/) [26] and miRDB (http://mirdb.org) [27].

\section{Bioinformatics analysis}

Based on DAVID 6.8 (https://david.ncifcrf.gov/home. jsp), we conducted the Gene Ontology (GO) and Kyoto Encyclopedia of Genes and Genomes (KEGG) analysis. GO analysis was used to identify the functional roles of circRNA-targeting genes in terms of cellular components, biological processes and molecular functions. KEGG analysis was performed to explore the pathways related to circRNA-targeting genes.

\section{Statistical analysis}

All data is described as mean \pm standard deviation (SD). All statistical analyses were carried out using SPSS statistical software version 18.0 (SPSS, Chicago, USA), and $P<0.05$ was considered statistically significant. CircRNA expression profiles in testicular tissue samples of the NOA and control group were analyzed by using paired $t$ test. CircRNAs that demonstrate fold changes $(\geq 2)$ were selected as being significantly differentially expression, and the false discovery rate (FDR) was calculated to correct the $P$ value of microarray analysis results. Correlations between the relative expression of circRNAs and their ceRNA were evaluated by Pearson's correlation method.

\section{Results}

\section{Differential expression of circRNAs between the control} and NOA testes

Hierarchical clustering picture revealed the circRNA expression profile in testicular tissues of NOA patients and the control (Fig. 1a). Box plots show that the distributions of circRNAs in both NOA and the control were nearly the same after normalization (Fig. 1b). The scatter plots showed the variation of circRNA expression between the NOA and the control group (Fig. 1c). The values of $\mathrm{X}$ and $\mathrm{Y}$ axes in the scatter-plot are the normalized signal values of the samples ( $\log 2$ scaled) or the averaged normalized signal values of groups of samples ( $\log 2$ scaled). The green lines are Fold Change Lines. The circRNAs above the top green line and below the bottom green line indicated more than 2.0 fold change of circRNAs between the two compared samples. CircRNAs were considered to have significantly differential expression if they were up- or down-regulated at least two-fold.

A total of 4169 human circRNAs were detected. Of them, 526 human circRNAs were identified as up- 
(a)

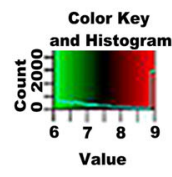

(d)

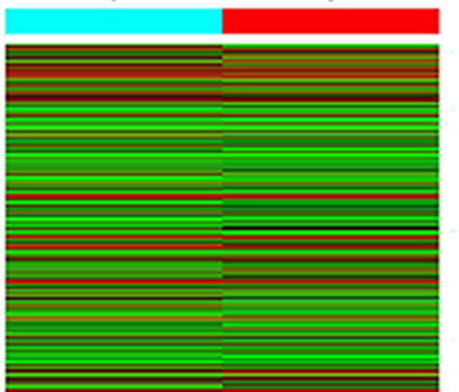

(c)
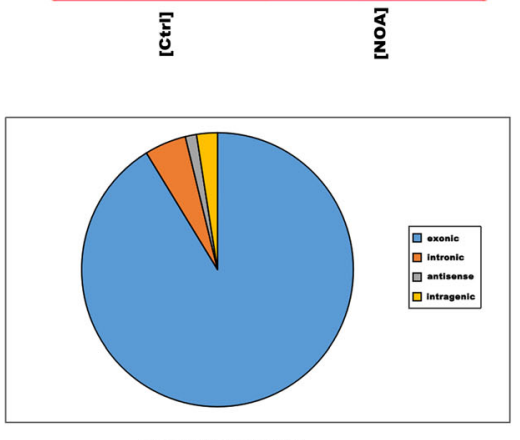

Up-regulation=526

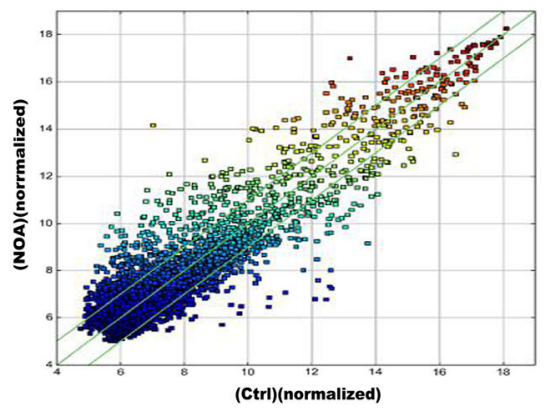

(b)
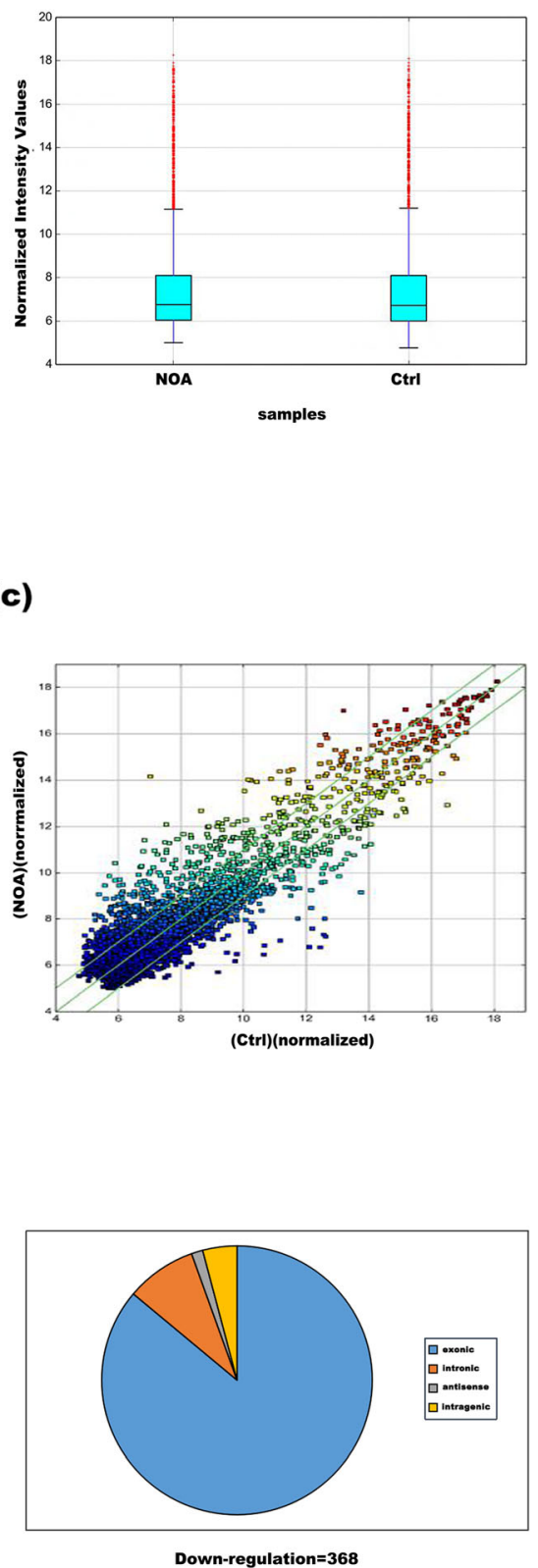

Fig. 1 Analysis of differentially expressed circRNA in testicular tissue of NOA patients. a Hierarchical clustering picture of all expressed circRNAs. "red color" represents high relative expression, and "green color" represents low relative expression. b Box plots show that the distributions of circRNAs in the control and NOA group samples were nearly the same after normalization. cThe scatter plots showed the variation of circRNA expression between the NOA and the control. The circRNAs in the located above the top green line and below the bottom green line indicated more than a 2.0-fold change of circRNAs. $\mathbf{d}$ The differentially expressed circRNAs based on the genomic origin were shown in the pie charts

regulated and 368 were down-regulated in testicular tissue of NOA patients compared to the controls $(\mathrm{aFC}>$ 2.0 and $P<0.05)$. According to the genomic origin of human circRNAs, the classification of the differentially expressed circRNAs was summarized in pie chart (Fig. 1d). Most of them belong to exonic circRNAs. In detail, the 526 up-regulated circRNAs consisted of 479 exonic, 26 intronic, 8 antisense and 13 intragenic. In addition, the 368 down- regulated circRNAs included 316 exonic, 31 intronic, 6 antisense and 15 intragenic (Fig. 1d).

\section{Validation of mircroarray data by using qRT-PCR}

To confirm the circRNA microarray results, qRT-PCR analysis was performed on 6 randomly selected differentially expressed circRNAs, including 3 up-regulated circRNAs (hsa_circ_0058058, hsa_circ_0008045 and hsa 
circ_0023313) and 3 down-regulated circRNAs (hsa circ_0061817, hsa_circ_0002023, and hsa_circ_0008533) in control and NOA group testicular tissue samples. The results indicated that the expression patterns of selected circRNAs were in consistent with microarray data (Fig. 2), in which hsa_circ_0023313 (Control $1.30 \pm 1.33$, NOA $16.46 \pm 2.81, P=0.002$ ), hsa_circ_0008045 (Control $1.00 \pm 0.32$, NOA $4.12 \pm 0.51, P=0.00035)$ and hsa circ_0058058 (Control $0.98 \pm 0.43$, NOA $16.93 \pm 1.48$, $P=0.0004)$ was up-regulated, and hsa_circ_0061817 (Control $1.04 \pm 0.24$, NOA $0.58 \pm 0.19, P=0.061$ ), hsa circ_0002023 (Control $1.00 \pm 0.29$, NOA $0.46 \pm 0.13, P=$ 0.01 ), and hsa_circ_0008533 (Control $0.99 \pm 0.26$, NOA $0.60 \pm 0.16, P=0.012)$ was down-regulated in NOA patients, compared with the control group.

\section{CircRNA/miRNA interaction analysis}

It has been demonstrated that circRNAs function as miRNA "sponges" that competitively suppress miRNA activity and further regulate the gene expression. To find the potential circRNA/miRNA interaction in NOA, one confirmed circRNA (hsa_circRNA_0023313) was selected for further bioinformatics analysis and prediction.

For hsa_circRNA_0023313, the most likely potential target miRNAs are hsa-miR-520d-3p, hsa-miR-373-3p,
hsa-miR-372-3p, hsa-miR-302c-3p and hsa-miR-130b$5 \mathrm{p}$. The sequence analysis of miRNA response elements (MREs) are shown in Fig. 3. "The 2D structure" demonstrated the MRE sequence, the target miRNA seed type and the 3' pairing sequence. The "Local AU" showed the AU content $30 \mathrm{nt}$ upstream and downstream seed sequence. The red bars represent $\mathrm{A} / \mathrm{U}$ and high accessibility, while the black bars represent $\mathrm{G} / \mathrm{C}$ and low accessibility of the seed. Furthermore, the accessibility extent is demonstrated by the height of the bar. The "Position" stands for the most likely relative MRE position on the linear presentation of hsa_circRNA_002313.

\section{Prediction of circRNA/miRNA/mRNA interaction network} The circRNA/microRNA/mRNA interaction network diagram (Fig. 4) based on the predicted target genes of hsa circRNA_0023313-targeting miRNAs (including hsa-miR520d-3p, hsa-miR-373-3p, hsa-miR-372-3p, hsa-miR302c-3p and hsa-miR-130b-5p) was drawn by Cytoscape (https://cytoscape.org/) [28].

\section{Go analysis and KEGG pathway analysis}

Go analysis and KEGG pathway analysis were used to predict the potential biological functions of hsa_circRNA_0023313.

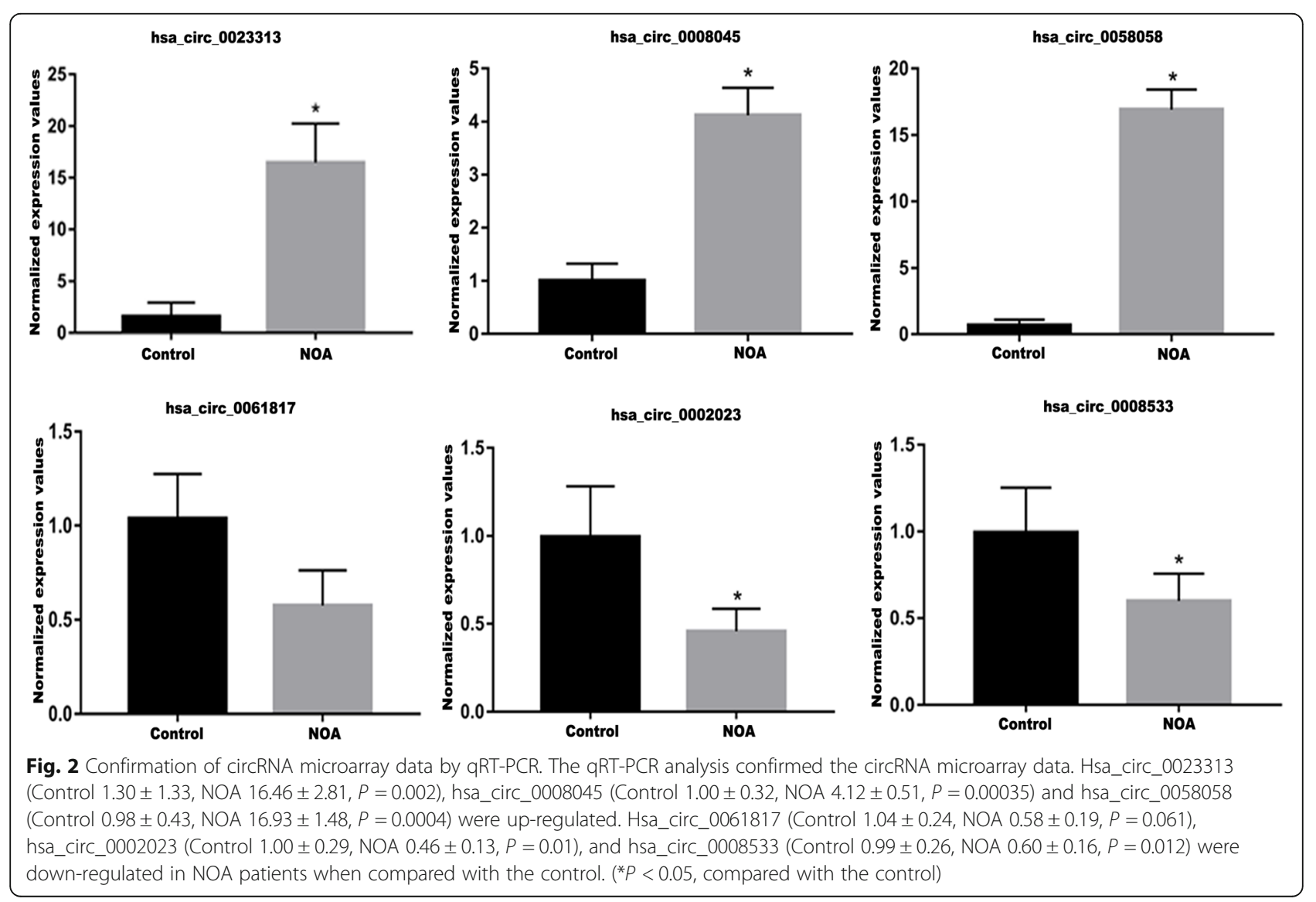


hsa-miR-520d-3p_vs_hsa_circRNA_0023313

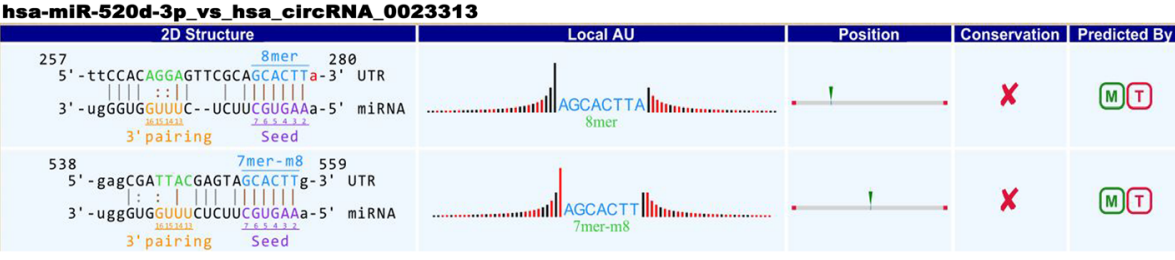

hsa-miR-373-3p_vs_hsa_circRNA_0023313

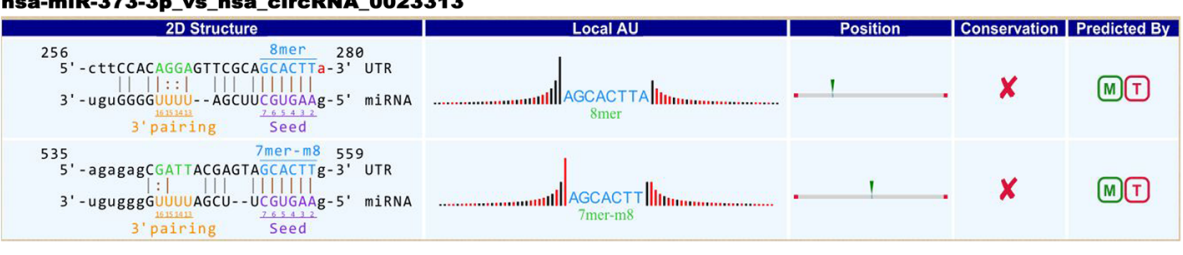

hsa-miR-372-3p_vs_hsa_circRNA_0023313

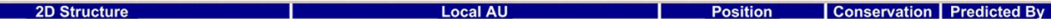

241.

3. - ugcGagu

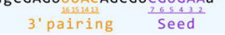

257. - ttCCACAGGAGTTCGCAGCACTTa- $3^{\circ}$. UTR

3'- ugcGaguiuaC-AGCGUCGUGAa- - 5' miRna

3'pairing seed

537. - agagcgattacgaGTAGGCACTT $53^{\circ}$ UTR

3. - ugcgaguuuacagCGUCGUGAAa- 5. miRNA

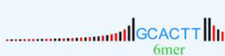

$x$

(il)

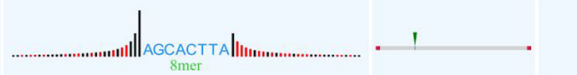

$x$

(M)

3'pairing Seed

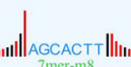

X M

hsa-miR-302c-3p_vs_hsa_circRNA_0023313

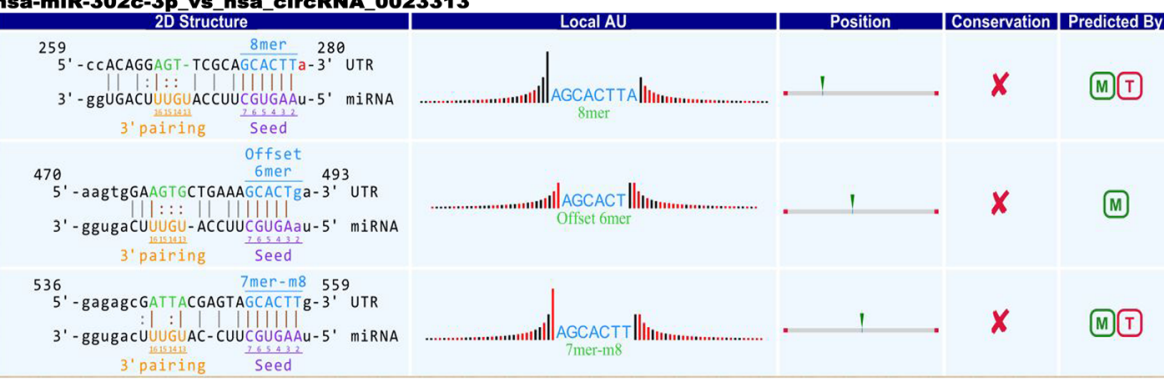

hsa-miR-130b-5p_vs_hsa_circRNA_0023313

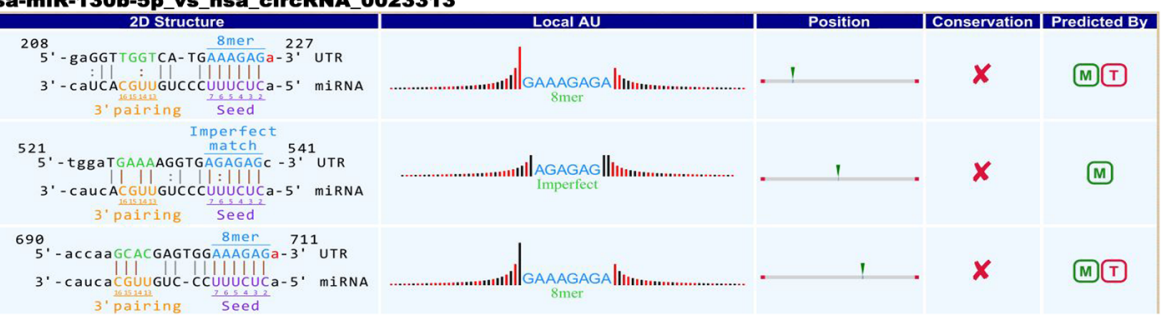

Fig. 3 Prediction of circRNA/miRNA interaction information of hsa_circRNA_0023313. The results of hsa_circRNA_0023313 bound to sites of hsamiR-520d-3p, hsa-miR-373-3p, hsa-miR-372-3p, hsa-miR-302c-3p and hsa-miR-130b-5p in 3'-UTR

As shown in Fig. 5, for hsa_circRNA_0023313, the cellular component analysis revealed that its target genes were mainly involved in cytoplasm, cytosol and autophagosome and autophagosome (Fig. 5a). The biological process analysis showed its target genes were mainly involved in positive regulation of transcription, DNA-templated, positive regulation of transcription from RNA polymerase II promoter and covalent chromatin modification etc (Fig. 5b). Moreover, the molecular function analysis indicated that hsa_circRNA_0023313 was mainly involved in ubiquitin-protein transferase activity, chromatin binding and ATP-binding and so on (Fig. 5c).

KEGG analysis revealed that the top five pathways related to hsa_circRNA_0023313 were endocytosis, meiosis, FoxO signaling pathway, Ubiquitin mediated proteolysis and AMPK signaling pathway (Fig. 5d).

\section{Discussion}

As far as we know, this is the first study to identify the comprehensive circRNAs expression pattern in testicular tissues of NOA patients. The microarray data revealed 


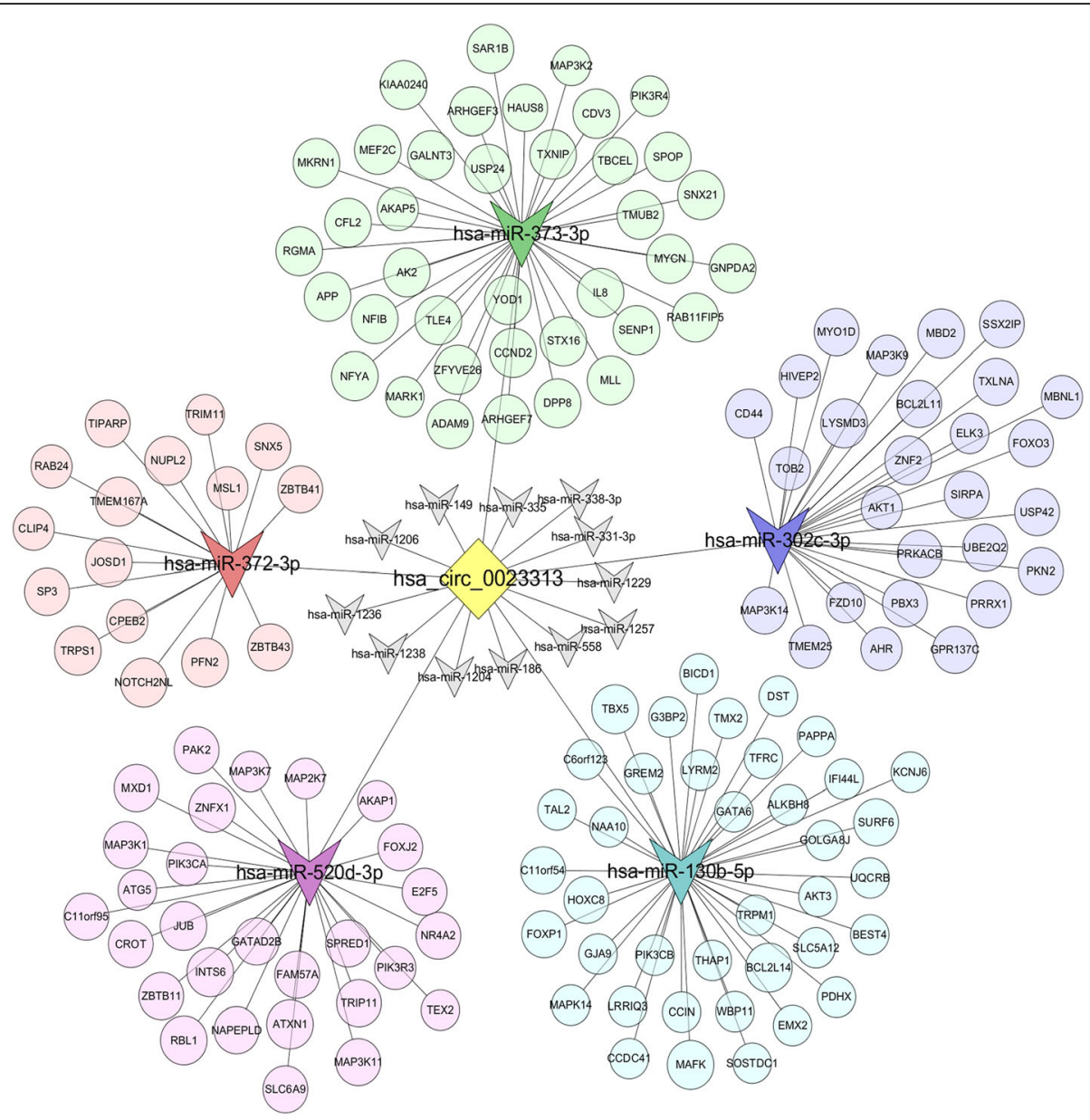

Fig. 4 CircRNA/miRNA/mRNA interaction network diagram based on the predicted target genes of hsa_circRNA_0023313-targeting miRNAs. The yellow square in the center stands for the hsa_circRNA_0023313. The triangles in different color represent potential target miRNAs for hsa_circRNA_0023313. The different color round stands for the potential corresponding target genes (mRNA) of hsa_circRNA_0023313-targeting miRNAs

that 368 circRNAs were down-regulated and 526 circRNAs were up-regulated $(\mathrm{aFC}>2.0$ and $P<0.05)$. These findings have been confirmed by qRT-PCR assays on randomly selected circRNAs, including hsa_circ_0023313, hsa circ_0058058, hsa_circ_0008045, hsa_circ_0061817, hsa_ circ_0002023, and hsa_circ_0008533. Further systemic bioinformatics analyses including the circRNA/miRNA/mRNA interaction network, GO and KEGG pathway analysis were used to predict the functions of differentially expressed circRNAs suggesting a potential important role of circRNAs in regulating spermatogenesis.

Spermatogenesis, the transformation of spermatogonia into sperm, is a well-orchestrated and precisely-regulated biological process which is strictly regulated by phasespecific gene expression [4, 29-31]. Noncoding RNAs such as microRNAs (miRNAs), PIWI-interacting RNAs (piRNAs) and long non-coding RNAs (lncRNAs) are important post-transcriptional regulators of gene expression in multiple stages of spermatogenesis [32, 33]. CircRNAs are a novel class of conserved endogenous noncoding
RNAs that could regulate gene expression [33]. It is probably the largest RNA families in human transcription [33]. Unlike linear RNAs, circRNAs form a covalently closed continuous loop and are highly represented in the eukaryotic transcriptome [13]. Thus, circRNAs are highly conserved and stability, therefore might be involved in more abundant functions [19]. Dong et al. reported that the expression of circRNAs in human testis is abundant, there are 15,996 circRNAs in normal human testis which participate the accurate gene expression regulations in spermatogenesis,, and their sequencing data were publicly in SRA database [33].

By using the circRNAs microarray and qRT-PCR analysis, we compared the circRNAs expression patterns in testicular tissues between NOA and the control. Our microarray results showed that 368 circRNAs were down-regulated and 526 circRNAs were up-regulated. To confirm the microarray data, 6 differentially expressed circRNAs were randomly selected for detection in 50 pairs of testicular tissues by qRT-PCR 

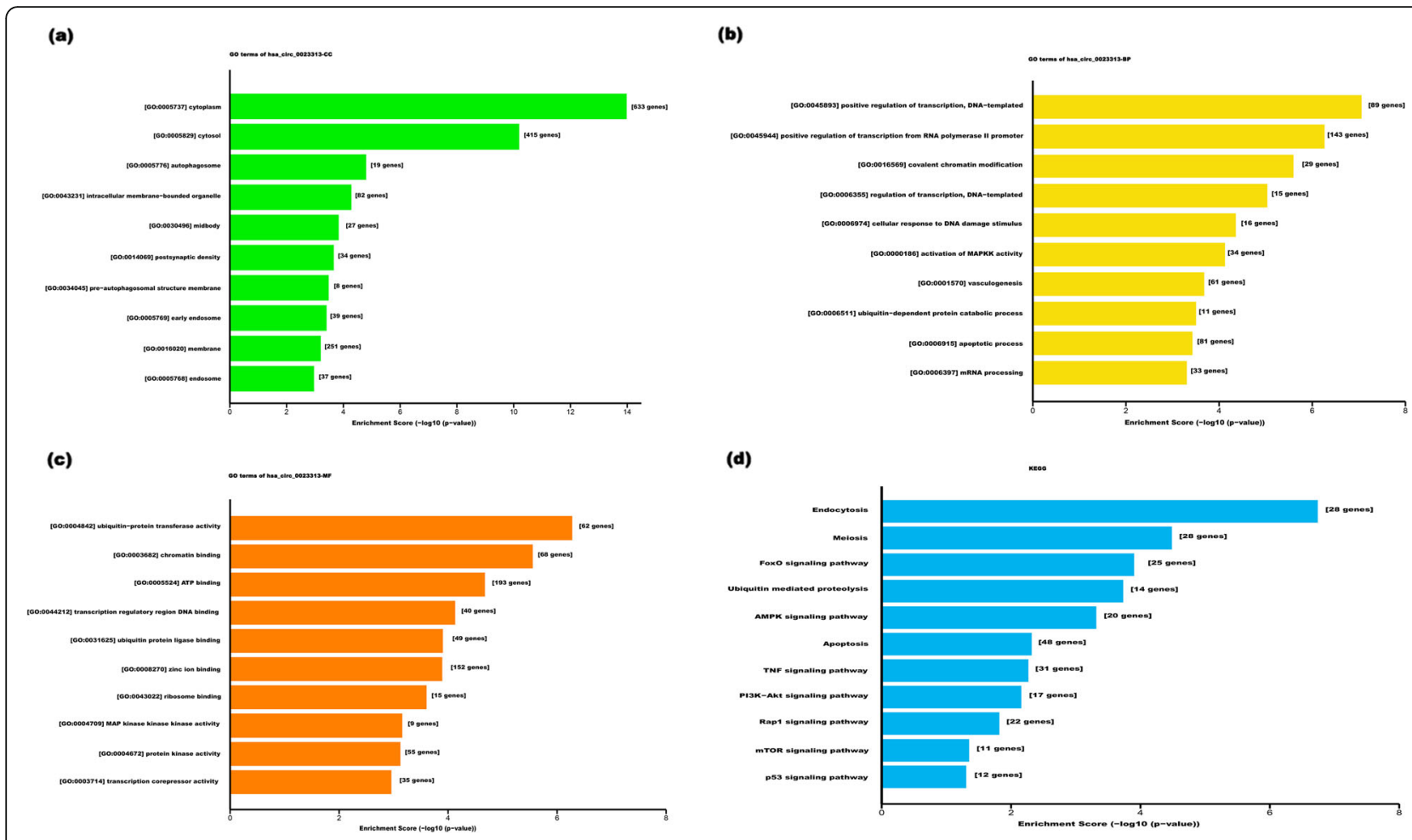

(d)

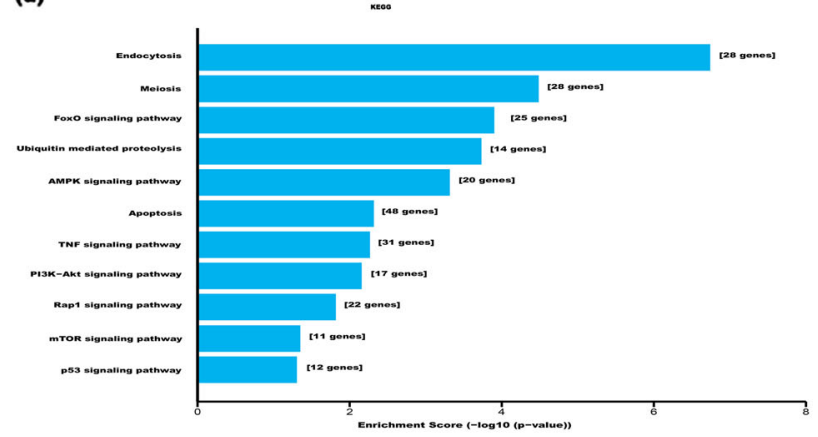

Fig. 5 Go analysis and KEGG pathway analysis for has_circ_0023313. a Cellular component analysis for has_circ_0023313 targeting genes; b Biological process analysis for has_circ_0023313 targeting genes; c Molecular function analysis for has_circ_0023313 targeting genes; d KEGG pathway analysis for has_circ_0023313

methods. The consistency between microarray data and qRT-PCR results further hinted that circRNAs might play important roles in regulating spermatogenesis. At the same time, we compared with the database (SRX2254041) of normal human testis circRNA deep sequence, the all of 6 circRNAs we selected were included in this database, and each of circRNA had changed [33]. Among these dysregulated circRNAs, the expression of hsa_circRNA_0023313 was dramatically enhanced in NOA patients, which indicated that it might play important roles in regulating spermatogenesis and potential biomarkers for the diagnosis, treatment of NOA.

CircRNA/miRNA/mRNA interaction network prediction provides a comprehensive understanding of the biological functions of hsa_circRNA_0023313. Our circRNA/miRNA interaction analysis demonstrated that the most likely potential target miRNA for hsa_circRNA_0023313 include hsa-miR-373-3p, hsa-miR-3723p, hsa-miR-520d-3p, hsa-miR-302c-3p and hsa-miR130b-5p. The study of Liu et al. showed that hsa-miR373 and hsa-miR-372 were dysregulated in the semen of infertile males with semen abnormalities, which might be associated with semen abnormalities in infertile males [34]. In addition, Syring et al. reported that serum hsamiR-373-3p and hsa-miR-372-3p levels were significantly increased in patients with testicular germ cell tumor compared to healthy individuals and patients with nonmalignant testicular disease [35]. The study of Hansen et al. found that the testis-specific circRNA, sexdetermining region $\mathrm{Y}$ (Sry), serves as a miR-138 sponge, suggesting that miRNA sponge effects achieved by circRNA formation are a general phenomenon [36]. It has been showed that circRNAs function as miRNA "sponges" that competitively suppress miRNA activity and further regulate the target gene expression, and also existed in the normal human testis, thereby contributing to the development of disease [14,33]. In the present study, hsa_circRNA_0023313 was up-regulated in NOA patients, which indicate that hsa_circRNA_0023313 might be inhibition of spermatogenesis by suppressing miRNA activity.

CircRNAs may compete with linear RNAs by binding miRNAs with miRNAs response elements (MREs), which strongly suppress miRNA activity and result in increased levels of miRNA target genes [36]. In our study, we found that putative target genes of hsa-miR-372-3p included autophagy relative gene such as RAB-24 [37] Increasing evidence suggests that autophagy play a critical role in the pathogenesis of male infertility [30, 38]. In addition, our data showed that putative target genes of hsa-miR-373-3p included ubiquitin specific protease gene such as USP24. Recent study also reported USP24 
is an AR-target gene, the increased expression of the USP24 gene was associated with the initiation of sexual development, which may be involved in the regulation of spermatogenesis in mice [39]. The hsa_circRNA_0023313 may increase the expression of these target genes through competitive binding with miRNA. We speculated that hsa_circRNA_0023313 may regulate spermatogenesis by hsa_circRNA_0023313/ miR-372-3p / RAB-24 pathway and/or hsa_circRNA_0023313/ miR-373-3p / USP-24 pathway, which highly reflects the role of ceRNA regulatory network. However, the verification experiment on detailed molecular mechanisms is needed in the future.

At the same time, Go analysis and KEGG pathway analysis were used to predict the potential biological functions of hsa_circRNA_0023313. The cellular component analysis revealed that the target genes of hsa_circRNA_0023313 were mainly involved in cytoplasm, cytosol and autophagosome. The biological process analysis showed its target genes were mainly take part in positive regulation of transcription, DNA-templated and positive regulation of transcription from RNA polymerase II promoter. The molecular function analysis indicated that it mainly focuses on ubiquitin-protein transferase activity, chromatin binding and ATP-binding and so on. KEGG analysis revealed that the top five pathways related to hsa_circRNA_0023313 were endocytosis, meiosis, FoxO signaling pathway, Ubiquitin mediated proteolysis and AMPK signaling pathway. All these data strongly indicate that hsa_circRNA_0023313 might be closely related to the initiation and progression of spermatogenesis.

\section{Conclusions}

In conclusion, this work illustrates for the first time that the comprehensive expression pattern of circRNAs in testicular tissues of NOA patients, indicating that circRNAs might play important roles in regulating spermatogenesis and it might be potential molecular targets for diagnosis and treatment of NOA. However, the exploration of molecular mechanism about the detailed role of circRNAs on spermatogenesis are still needed in the future.

\section{Acknowledgments}

The authors thank all the subjects who participated in this study. In addition, they thank all the technologists for their cooperation and contribution to this study.

\section{Authors' contributions}

$P G, J Z$, and $L Z$ designed the project with guidance from DXZ. LZ provided the testicular tissue biopsies and patients clinical examination results. GP conducted bioinformatics analysis. PG, YXL, and JW performed the experiments, with the technical support of MQL. The manuscript was written by DXZ with contributions from PG and JZ. The diagrammatic illustrations were created by DXZ. All authors read and approved the final manuscript.

\section{Funding}

This research was financially supported by National Natural Science Funding of China (No. 81673224; 81273018; 30700654), Natural Science Funding of Shaanxi Province (No. 2019JM029; 2018JM7135; 2015JM8436), Science Funding of Health Department, Shaanxi Province (2012D58), the Fundamental Research Funds for the Central University (XJJ 2011024) and the Project of Independent Innovative Experiment for Postgraduates in medicine in Xi'an Jiaotong University (YJSCX-2017-007 and YJSCX2018-011).

\section{Availability of data and materials}

The dataset supporting the conclusions of this article is included within the article.

Ethics approval and consent to participate

All procedures performed in this study involving were in accordance with the ethical standards of the Institutional Medical Ethics Committee of Xi'an Jiaotong University.

\section{Consent for publication}

Not applicable.

\section{Competing interests}

The authors declare that they have no competing interests.

\section{Author details}

${ }^{1}$ Department of Pathology, Medical School, Xi'an Jiaotong University, Xi'an 710061, China. ${ }^{2}$ Research Center of Reproductive Medicine, Medical School, Xi'an Jiaotong University, Xi'an 710061, China. ${ }^{3}$ Reproductive Center Medicine, Maternal and child care Hospital of Shaanxi Province, Xi'an 710063, China. ${ }^{4}$ Obstetrics and Gynecology Department, Xi'an Angel Women's \& children's Hospital, Xi'an 710077, China. ${ }^{5}$ Obstetrics and Gynecology Department, Maternal and child care Hospital of Shaanxi Province, Xi'an 710063, China.

Received: 8 August 2019 Accepted: 5 November 2019

Published online: 27 November 2019

References

1. Fainberg J, Kashanian JA. Recent advances in understanding and managing male infertility. F1000Research. 2019;8:1-8.

2. Khourdaji I, Lee H, Smith RP. Frontiers in hormone therapy for male infertility. Transl Androl Urology. 2018;7:S353-353\$366.

3. Ferlin A, Foresta C. New genetic markers for male infertility. Curr Opin Obstet Gynecol. 2014;26:193-8.

4. Cannarella R, Condorelli RA, Duca Y, La Vignera S, Calogero AE. New insights into the genetics of spermatogenic failure: a review of the literature. Hum Genet. 2019;138:125-40.

5. Okada H, Tajima A, Shichiri K, Tanaka A, Tanaka K, Inoue I. Genome-wide expression of azoospermia testes demonstrates a specific profile and implicates ART3 in genetic susceptibility. PLoS Genet. 2008;4:e26.

6. Nakamura S, Miyado M, Saito K, Katsumi M, Nakamura A, Kobori Y, Tanaka Y, Ishikawa H, Yoshida A, Okada H, Hata K, Nakabayashi K, et al. Nextgeneration sequencing for patients with non-obstructive azoospermia: implications for significant roles of monogenic/oligogenic mutations. Andrology. 2017;5:824-31.

7. Gu X, Li H, Chen X, Zhang X, Mei F, Jia M, Xiong C. PEX10, SIRPA-SIRPG, and SOX5 gene polymorphisms are strongly associated with nonobstructive azoospermia susceptibility. J Assist Reprod Genet. 2019;36:759-68.

8. Bernie AM, Shah K, Halpern JA, Scovell J, Ramasamy R, Robinson B, Schlegel $\mathrm{PN}$. Outcomes of microdissection testicular sperm extraction in men with nonobstructive azoospermia due to maturation arrest. Fertil Steril. 2015;104: 569-573.e1.

9. Arafat M, Har-Vardi I, Harlev A, Levitas E, Zeadna A, Abofoul-Azab M, Dyomin V, Sheffield VC, Lunenfeld E, Huleihel M, Parvari R. Mutation in TDRD9 causes non-obstructive azoospermia in infertile men. J Med Genet. 2017;54:633-9.

10. Liu W, Gao X, Yan L, Liu H, Yu R, Zhao S, Ma J. Analysis of CDK2 mutations in Chinese men with non-obstructive azoospermia who underwent testis biopsy. Reprod BioMed Online. 2018;36:356-60. 
11. Oka S, Shiraishi K, Matsuyama H. Effects of human chorionic gonadotropin on testicular interstitial tissues in men with non-obstructive azoospermia. Andrology. 2017:5:232-9.

12. Memczak S, Jens M, Elefsinioti A, Torti F, Krueger J, Rybak A, Maier L, Mackowiak SD, Gregersen LH, Munschauer M, Loewer A, Ziebold U, et al. Circular RNAs are a large class of animal RNAs with regulatory potency. Nature. 2013;495:333-8.

13. Hentze MW, Preiss T. Circular RNAs: splicing's enigma variations. EMBO J. 2013:32:923-5.

14. Qu S, Yang X, Li X, Wang J, Gao Y, Shang R, Sun W, Dou K, Li H. Circular RNA: A new star of noncoding RNAs. Cancer Lett. 2015;365:141-8.

15. Liu W, Zhang J, Zou C, Xie X, Wang Y, Wang B, Zhao Z, Tu J, Wang X, Li H, Shen J, Yin J. Microarray expression profile and functional analysis of circular RNAs in osteosarcoma. Cell Physiol Biochem. 2017;43:969-85.

16. Jiang S, Guo C, Zhang W, Che W, Zhang J, Zhuang S, Wang Y, Zhang Y, Liu B. The integrative regulatory network of circRNA, microRNA, and mRNA in atrial fibrillation. Front Genet. 2019;10:526.

17. Burd CE, Jeck WR, Liu Y, Sanoff HK, Wang Z, Sharpless NE. Expression of linear and novel circular forms of an INK4/ARF-associated non-coding RNA correlates with atherosclerosis risk. PLoS Genet. 2010;6:e1001233.

18. Li P, Chen S, Chen H, Mo X, Li T, Shao Y, Xiao B, Guo J. Using circular RNA as a novel type of biomarker in the screening of gastric cancer. Clin Chim Acta. 2015;444:132-6.

19. Yao JT, Zhao SH, Liu QP, Lv MQ, Zhou DX, Liao ZJ, Nan KJ. Over-expression of CircRNA 100876 in non-small cell lung cancer and its prognostic value. Pathol Res Pract. 2017:213:453-6.

20. Vloeberghs $V$, Verheyen $G$, Haentjens P, Goossens A, Polyzos NP, Tournaye H. How successful is TESE-ICSI in couples with non-obstructive azoospermia. Hum Reprod. 2015;30:1790-6.

21. Sánchez-Álvarez J, Cano-Corres R, Fuentes-Arderiu X. A complement for the WHO Laboratory manual for the examination and processing of human semen (first edition, 2010). EJIFCC. 2012;23:103-6.

22. Björndahl L, Barratt $C L$, Mortimer D, Jouannet P. 'How to count sperm properly': checklist for acceptability of studies based on human semen analysis. Hum Reprod. 2016;31:227-32.

23. World Health Organisation. WHO Laboratory Manual for the Examination and Processing of Human Semen. 5th ed. Geneva: World Health Organisation; 2010.

24. Pasquinelli AE. MicroRNAs and their targets: recognition, regulation and an emerging reciprocal relationship. Nat Rev Genet. 2012;13:271-82.

25. Enright AJ, John B, Gaul U, Tuschl T, Sander C, Marks DS. MicroRNA targets in drosophila. Genome Biol. 2003:5:R1.

26. Zhang J, Fan D, Jian Z, Chen GG, Lai PB. Cancer specific long noncoding RNAs show differential expression patterns and competing endogenous RNA potential in hepatocellular carcinoma. PLoS One. 2015;10:e0141042.

27. Wong $N$, Wang $X$. miRDB: an online resource for microRNA target prediction and functional annotations. Nucleic Acids Res. 2015;43:D146-52.

28. Shannon P, Markiel A, Ozier O, Baliga NS, Wang JT, Ramage D, Amin N, Schwikowski B, Ideker T. Cytoscape: a software environment for integrated models of biomolecular interaction networks. Genome Res. 2003;13:2498-504.

29. Fang J, Li DH, Yu XQ, Lv MQ, Bai LZ, Du LZ, Zhou DX. Formaldehyde exposure inhibits the expression of mammalian target of rapamycin in rat testis. Toxicol Ind Health. 2016;32:1882-90.

30. Yin J, Ni B, Tian ZQ, Yang F, Liao WG, Gao YQ. Regulatory effects of autophagy on spermatogenesis. Biol Reprod. 2017:96:525-30.

31. Griswold MD. Spermatogenesis: the commitment to meiosis. Physiol Rev. 2016:96:1-17.

32. Mukherjee A, Koli S, Reddy KV. Regulatory non-coding transcripts in spermatogenesis: shedding light on 'dark matter'. Andrology. 2014;2:360-9.

33. Dong WW, Li HM, Qing XR, Huang DH, Li HG. Identification and characterization of human testis derived circular RNAs and their existence in seminal plasma. Sci Rep. 2016:6:39080.

34. Liu T, Cheng W, Gao Y, Wang H, Liu Z. Microarray analysis of microRNA expression patterns in the semen of infertile men with semen abnormalities. Mol Med Rep. 2012;6:535-42.

35. Syring I, Bartels J, Holdenrieder S, Kristiansen G, Müller SC, Ellinger J. Circulating serum miRNA (miR-367-3p, miR-371a-3p, miR-372-3p and miR-373-3p) as biomarkers in patients with testicular germ cell cancer. J Urol. 2015;193:331-7.

36. Hansen TB, Jensen TI, Clausen BH, Bramsen JB, Finsen B, Damgaard CK, Kjems J. Natural RNA circles function as efficient microRNA sponges. Nature. 2013;495:384-8.
37. Toh H, Nozawa T, Minowa-Nozawa A, Hikichi M, Nakajima S, Aikawa C, Nakagawa I. Group a Streptococcus modulates RAB1- and PIK3C3 complexdependent autophagy. Autophagy. 2019;14:1-13.

38. Han SP, Zhou DX, Lin P, Qin Z, An L, Zheng LR, Lei L. Formaldehyde exposure induces autophagy in testicular tissues of adult male rats. Environ Toxicol. 2015;30:323-31.

39. Hu QY, Deng Q, Zhang JW, Zhi F, Sun R, Liang H. Expression characteristics of the USP24 gene in the mouse testis during spermatogenesis. Zhonghua Nan Ke Xue. 2017:23:963-8.

\section{Publisher's Note}

Springer Nature remains neutral with regard to jurisdictional claims in published maps and institutional affiliations.
Ready to submit your research? Choose BMC and benefit from:

- fast, convenient online submission

- thorough peer review by experienced researchers in your field

- rapid publication on acceptance

- support for research data, including large and complex data types

- gold Open Access which fosters wider collaboration and increased citations

- maximum visibility for your research: over $100 \mathrm{M}$ website views per year

At BMC, research is always in progress.

Learn more biomedcentral.com/submissions 